\title{
Modulatory role of ketogenic diet on neuroinflammation; a possible drug naïve strategy to treatment of Parkinson's disease
}

Sheyda Shaafi ${ }^{1}$, Javad Mahmoudi ${ }^{1}$, Safa Najmi ${ }^{1}$, Saeed Sadigh-Eteghad ${ }^{1}$, Mehdi Farhoudi ${ }^{1}$, Hamed Aliasgharpour ${ }^{1 *}$

1. Neurosciences Research Center (NSRC), Tabriz University of Medical Sciences, Tabriz, Iran

\begin{abstract}
Parkinson's disease is the second common neurological disease associated with elderly. Neuroinflammation contributes in neuronal death in Parkinson's disease (PD). The ketogenic diet (KD) is low in carbohydrates, adequate in protein, and high in fat, has been used in intractable epilepsy and seems to be effective in neurologic disorders. The implication of Neuroinflammation in PD has been emphasized and there is evidence show that KD may provide advantages to reduce neuroinflammatory adverse effects. Based on this surmise, it seems that KD could reverse some neuronal injuries of PD.
\end{abstract}

Keywords: Ketogenic diet, Neuroinflammation, Parkinson's disease

\section{Introduction}

Parkinson's disease (PD) is a neurodegenerative disorder affects elderly older than 65 years and characterized by irreversible loss of dopaminergic (DAergic) neurons in the substantia nigra pars compacta (SNcP) and their terminal in the striatum. In PD, destruction of DAergic neurons leads to reduction of striatal dopamine levels by $70 \%$ and appearance of hypokinetic motor signs such as akinesia, tremor at rest, muscle rigidity, and postural instability $(1,2)$. The exact mechanisms underlying of PD have not been understood, but numerous studies suggest that neuroinflammation,(3) apoptosis (4) and oxidative stress may play role in PD. There isn't

any approved treatment for delaying of PD neuronal damages and current therapies only manage its symptoms. Given this, development of novel strategies to reduction of PD insults is a high priority. Since, chronic pharmacotherapy for PD is accompanied by some adverse effects (2), hence application of non-therapeutic approaches in PD is of interest. In this paper we aimed to focus on the role of KD and neuroinflammation in PD.

\section{Ketogenic Diet}

The ketogenic diet (KD) is a dietary regimen for producing of continuous ketonemia.

It was introduced for first time in 1920 contains high-fat, low-carbohydrate, and low-

\section{Corresponding author:}

Hamed Aliasgharpour

Neurosciences Research Center (NSRC), Tabriz University of Medical Sciences, Tabriz, Iran

Email: dr.asgharpour@yahoo.com

Receive date: 2015-06-29| Accept date: 2015-07-22| Publish date: 2015-08-04

DOI: 10.7575/aiac.abcmed.15.03.04.09

\section{A. I}


protein and is used to remission of drug resistance epilepsies.5 KD reduces gluconeogenesis from amino acids and activates hepatic ketogenesis through conversation of fatty acids and elevates circulating levels of major ketone bodies ( $\beta$ hydroxybutyrate) $(6,7)$. Under particular circumstances such as fasting, sever physical activity and hypometabolism, ketons can substitute for glucose and pyruvate as an auxiliary fuel source (8). These molecule, can penetrate the brain blood barrier (BBB) via their transporters such as monocarboxylic acids which are located on the endothelial cells of the BBB (7).

In the brain, ketone bodies (KBs) can be taken up by both neuronal (9) and nonneuronal cells, (7) then are consumed by the neurons in a concentration-dependent fashion. KBs provide nearly $70 \%$ of brain's energy expenditure (9). Within the mitochondria, succinyl-CoA: 3-ketoacid CoA transferase (SCOT) and acetyl-CoA acetyltransferase 1 (ACAT1) oxidizes KBs to the acetyl-CoA10 and then it enters to the Krebs cycle (11) to ATP production. There is evidence that show, induction of ketonemia by ketogenic diet may improve some of neurological conditions such as, cognitive deficits, (12) spinal cord injury, (6) ischemic stroke,(5) amyotrophic lateral sclerosis (13) and PD (11). Given this, present paper aims to review possible mechanisms underlying of KD anti-PD effects.

\section{Neuroinflammtion}

\section{A. Proinflammatory cytokines and PD}

Accumulating data from epidemiological, post mortem findings and animal model of PD have demonstrated that neuroinflammation has role in pathogernesis of PD (14-16) Within the brain, neuroinflammtory responses are mediated through glial cells (17). Indeed, neuronal insults and some of some neurotoxins such as 6-hydroxydopamie (6-OHDA) drive these cells from the normal form to the activated condition and activated microglia known as a biomarker of neuroinflammation $(3,16)$. It is important to note, that 6 -OHDA as neurotoxin is used to modeling of PD in rats and its able to resemble some molecular and behavioral alterations seen in PD (17). Recently, sharifi et al., described the role of neuroinflammation in development of neurological impairments in 6-OHDA-lesioned parkinsonian rats. Measuring cerebrospinal fluid levels of pro-inflammatory cytokines, they have shown that activation of microglial cells, is accompanied by increasing levels of proinflammatory cytokines such as TNF- $\alpha$ and IL- 6 and modulation of microglial cells activity leads to attenuation of neuroinflammation and motor signs of PD (16).

Moreover, activated glial cells release reactive oxygen species (ROS) to cope with neurotoxic injuries. However, ill control of these molecules may results in oxidative stress, mitochondrial dysfunction and apoptosis mediated neuronal cell destruction (18). In addition to 6-OHDA, 1-methyl-4-phenol-1,2,5,6tetrahydropyridine (MPTP)-induced mice model of PD remains as a reliable tool to investigate of PD pathogenesis. Findings from MPTP- treated mice show that KD through modulation of microglial cell activation (19) mediates neuroprotective effects and improves behavioral outcomes of parkinsonian mice. Moreover, following systemic administration of MPTP, loss of tyrosine positive immunoreactive cells happens in nigrostriatal pathways. Immunohistochemical evidence also has shown that KD protects these neurons from the neurotoxic effects of MPTP (19) Beside this, Xu et al., studies on the microglia cell line, BV-2 cells, showed that, $\beta$-hydroxybutyrate, major component of ketone bodies, through inhibition of microglial activation prevents Lipopolysaccharide-induced inflammatory responses. They propose that, this molecule 
decreases expression and releasing of TNF- $\alpha$, IL-6 and IL-1 from the activated glial cells and increased cell viability in this invitro model of neuroinflammation (20). As depicted in figure 1 , this data shows that KD may have regulatory role on the neuroinflammtion in PD brain.

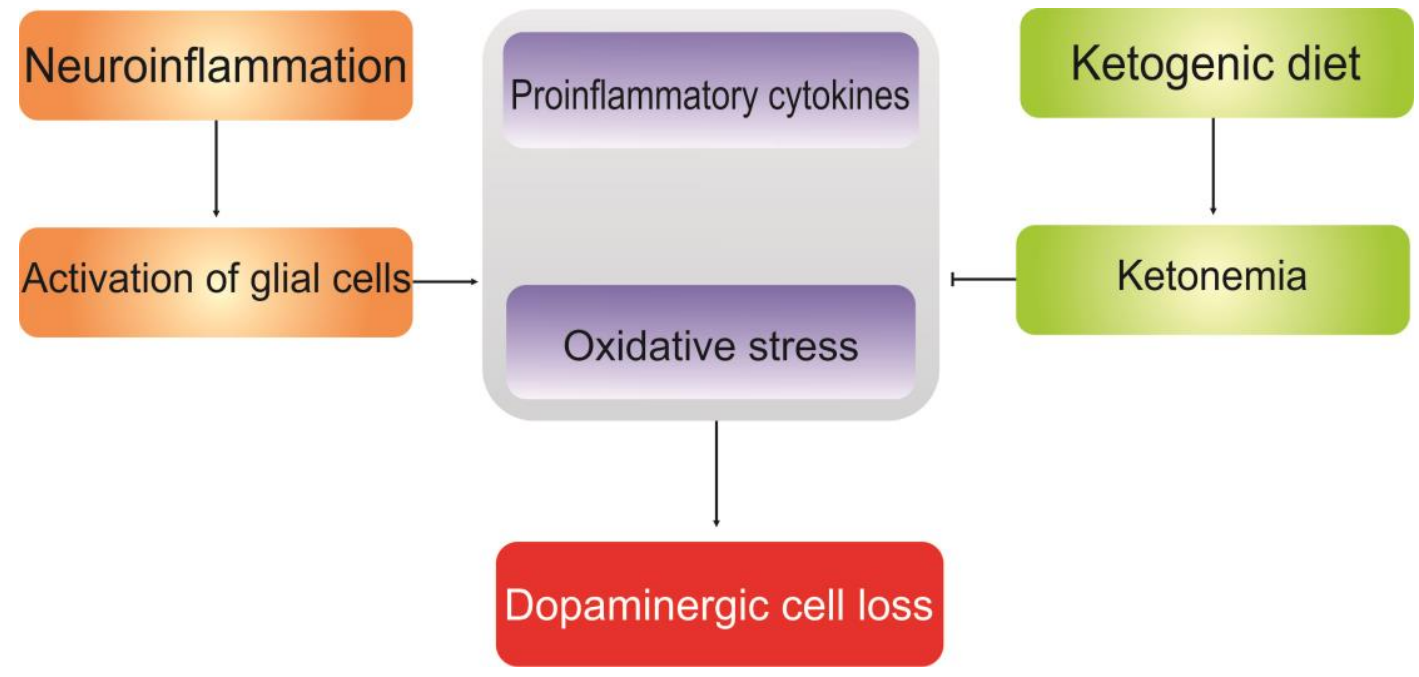

Figure 1: The implication of neuroinflammation and the effect of KD on DAergic cell loss in PD

\section{B. Oxidative stress and PD}

As mentioned, activated glial cells also provoke occurrence of oxidative stress in PD. It is propose that activated microglial cells through releasing of different ROS causes destruction of vulnerable neurons. DAergic neurons in the $\mathrm{SNpC}$ are susceptible to oxidative stress condition. This originates from these neurons high oxygen expenditure as well as lack of adequate amount of endogenous anti-oxidants enzymes in this region (21). Given this, application of molecules to reduce both microglial activation and oxidative stress for reduction of PD signs is of interest. Invivo findings show that, $\beta$-Hydroxybutyrate through attenuation of $\mathrm{H} 2 \mathrm{O} 2$, increases viability of $\mathrm{PC} 12$ cells (20). Notably, PC12 cell is obtained from the rat adrenal pheochromocytoma cell line (22) and widely used to assessment of some cellular and molecular aspects of neurodegenerative disorders (23). It has been suggested that, $\mathrm{H} 2 \mathrm{O} 2$ activates key enzymes of apoptotic cascades (i.e. caspase-3) and decreases endogenous anti-oxidants content such as glutathione in PC12 cell model of PD, hence it promotes apoptotic cell death. According to cheng et al., study, treatment of $\mathrm{H} 2 \mathrm{O} 2$ intoxicated $\mathrm{PC} 12$ cells by $\beta$ Hydroxybutyrate, ameliorate both oxidative stress and activation of apoptosis cascade, confirming that, ketogenic diet (figure 1 ) is able to augment anti-oxidant ability of neuronal cells (20).

\section{Final remark}

Neuroinflammation exerts its own neuronal injuries through activation of glial cell activation, and activated glial cells release different neurotoxic molecules such as proinflammatory cytokines and ROS which result in neuronal destruction. KD guards neurons by inhibition of oxidative stress and subsequent apoptotic cell death, but its exact neuroprotective mechanisms is poorly understood and reliable investigations must be designed to reveal its anti-PD mechanisms. 


\section{References}

1. Mahmoudi J, Mohajjel Nayebi A, Reyhani-Rad S, Samini M. Fluoxetine improves the effect of levodopa on 6-hydroxy dopamine-induced motor impairments in rats. Advanced pharmaceutical bulletin 2012;2(2):149-55.

2. Mahmoudi J, Nayebi AM, Samini M, Reyhani-Rad S, Babapour V. Buspirone improves the anti-cataleptic effect of levodopa in 6-hydroxydopamine-lesioned rats. Pharmacological reports : PR 2011;63(4):908-14.

3. Sharifi H, Nayebi AM, Farajnia S, Haddadi R. Effect of Chronic Administration of Buspirone and Fluoxetine on Inflammatory Cytokines in 6-Hydroxydopamine-lesioned Rats. Drug research 2014.

4. Winklhofer KF, Haass C. Mitochondrial dysfunction in Parkinson's disease. Biochimica et biophysica acta 2010;1802(1):29-44.

5. Shaafi S, Mahmoudi J, Pashapour A, Farhoudi M, Sadigh-Eteghad S, Akbari H. Ketogenic Diet Provides Neuroprotective Effects against Ischemic Stroke Neuronal Damages. 2014.

6. Streijger F, Plunet WT, Lee JH, Liu J, Lam CK, Park S, et al. Ketogenic diet improves forelimb motor function after spinal cord injury in rodents. PloS one 2013;8(11):e78765.

7. Vizuete AF, De Souza DF, Guerra MC, Batassini C, Dutra MF, Bernardi C, et al. Brain changes in BDNF and S100B induced by ketogenic diets in Wistar rats. Life sciences 2013;92(17):923-8.

8. Prins ML. Cerebral metabolic adaptation and ketone metabolism after brain injury. Journal of cerebral blood flow and metabolism : official journal of the International Society of Cerebral Blood Flow and Metabolism 2008;28(1):1-16.

9. Roy M, Nugent S, Tremblay-Mercier J, Tremblay S, Courchesne-Loyer A, Beaudoin JF, et al. The ketogenic diet increases brain glucose and ketone uptake in aged rats: a dual tracer PET and volumetric MRI study. Brain research 2012;1488:14-23.

10. Yao J, Chen S, Mao Z, Cadenas E, Brinton RD. 2-Deoxy-D-glucose treatment induces ketogenesis, sustains mitochondrial function, and reduces pathology in female mouse model of Alzheimer's disease. PloS one 2011;6(7):e21788.

11. Gasior M, Rogawski MA, Hartman AL. Neuroprotective and disease-modifying effects of the ketogenic diet. Behavioural pharmacology 2006;17(5-6):431-9.

12. Van Der Auwera I, Wera S, Van Leuven F, Henderson ST. A ketogenic diet reduces amyloid beta 40 and 42 in a mouse model of Alzheimer's disease. Nutrition \& Metabolism 2005;2:28-.

13. Zhao Z, Lange DJ, Voustianiouk A, Macgrogan D, Ho L, Suh J, et al. A ketogenic diet as a potential novel therapeutic intervention in amyotrophic lateral sclerosis. BMC neuroscience 2006;7(1):29.

14. Hirsch EC, Vyas S, Hunot S. Neuroinflammation in Parkinson's disease. Parkinsonism \& related disorders 2012;18 Suppl 1:S210-2.

15. Hunot S, Hirsch EC. Neuroinflammatory processes in Parkinson's disease. Annals of neurology 2003;53 Suppl 3:S4958; discussion S-60.

16. Sharifi H, Mohajjel Nayebi A, Farajnia S. 8-OH-DPAT (5-HT1A agonist) Attenuates 6-Hydroxy- dopamine-induced catalepsy and Modulates Inflammatory Cytokines in Rats. Iranian Journal of Basic Medical Sciences 2013;16(12):12705.

17. Meredith GE, Sonsalla PK, Chesselet M-F. Animal models of Parkinson's disease progression. Acta neuropathologica 2008;115(4):385-98.

18. Khorrami A, Ghanbarzadeh S, Mahmoudi J, Nayebi AM, Maleki-Dizaji N, Garjani A. Investigation of the Memory Impairment in Rats Fed with Oxidized-Cholesterol-Rich Diet Employing Passive Avoidance Test. Drug research 2014.

19. Yang X, Cheng B. Neuroprotective and anti-inflammatory activities of ketogenic diet on MPTP-induced neurotoxicity. Journal of molecular neuroscience : MN 2010;42(2):145-53.

20. Cheng B, Lu H, Bai B, Chen J. d- $\beta$-Hydroxybutyrate inhibited the apoptosis of PC12 cells induced by $\mathrm{H} 2 \mathrm{O} 2$ via inhibiting oxidative stress. Neurochemistry International 2013;62(5):620-5. 
21. Floyd RA. Antioxidants, Oxidative Stress, and Degenerative Neurological Disorders. Proceedings of the Society for Experimental Biology and Medicine 1999;222(3):236-45.

22. Westerink RHS, Ewing AG. The PC12 cell as model for neurosecretion. Acta physiologica (Oxford, England) 2008;192(2):273-85.

23. Sadigh-Eteghad S, Talebi M, Farhoudi M, Mahmoudi J, Reyhani B. Effects of Levodopa loaded chitosan nanoparticles on cell viability and caspase-3 expression in PC12 neural like cells. Neurosciences 2013;18(3): 281-3. 